\title{
Lista de espécies de aranhas da região central do Rio Grande do Sul - Família Araneidae
}

LEOCADIA FALKEMBERG INDRUSIAK

Regina Gressler Buss

\section{Resumo}

É apresentada uma lista de 58 espécies de aranhas da Família Araneidae que ocorrem na região central do Estado do Rio Grande do Sul, Brasil. É feita também a primeira citação de ocorrência, para o Estado, de Alpaida lanei Levi, 1998, Alpaida scriba (MelloLeitão, 1940) e Araneus sicki Levi, 1991. 


\section{Abstract}

A list of 58 spiders (Family Araneidae) from the central region of Rio Grande do Sul State (Brazil) is presented. It is also reported for the first time the occurrence of Alpaida lanei Levi, 1998, Alpaida scriba (Mello-Leitão, 1940) and Araneus sicki Levi, 1991 for this region.

\section{Introdução}

O objetivo deste trabalho é registrar a ocorrência de 58 espécies da Família Araneidae na região central do Rio Grande do Sul. A zona em questão é uma região de tensão ecológica (Leite \& Klein, 1990, cit apud Berriel, 2001) por estar localizada na faixa de transição entre o Domínio da Savana e o da Floresta Estacional Decidual; além disso, abrange uma área de grande importância faunística, qual seja a zona de transição entre o Planalto Meridional Brasileiro e a Depressão Central do Rio Grande do Sul, ponto de encontro de dois grandes domínios zoogeográficos neotropicais, o Guiano-Brasileiro e o Andino-Patagônico (Lema, 1984).

Esta é a primeira lista de aranhas da Família Araneidae publicada para esta região do Rio Grande do Sul. Quando da publicação do "Inventário das aranhas arborícolas de três regiões de Santa Maria, RS, Brasil" (Indrusiak \& Kotzian,1998) foi apresentada uma lista provisória arrolando 20 famílias, das quais a mais representativa, tanto em número de indivíduos quanto em diversidade, foi a Família Araneidae, representada, naquele trabalho, por 12 espécies, incluídas em 15 gêneros.

Berriel (2001) também constatou ser a família Araneidae a mais representativa dentre as 16 que detectou no levantamento que fez em plantas de caraguatá (Eryngium horridum Malme, 1904; Umbelliferae) em uma área de campo em Santa Maria, RS.

Buss (1993), pesquisando aranhas arborícolas nas matas-galeria de sangas e arroios de Cachoeira do Sul, RS, demonstrou que também naquela região Araneidae foi o grupo mais representativo dentre 18 famílias; nesse trabalho foram mencionados 21 gêneros, com 7 espécies determinadas.

Além disso, o material depositado, já parcialmente tombado, na coleção araneológica do Departamento de Biologia da Universidade Federal de Santa Maria, e que cobre um período de aproximadamente quinze anos de pesquisa de campo na região em ques- 
tão, demonstra a predominância da Família Araneidae, com cerca de $40 \%$ do total do acervo.

Todas estas razões, aliadas à disponibilidade de especialistas em Araneidae que colaboraram neste trabalho, possibilitando que a maior parte do material fosse determinado a nível de espécie, motivaram a publicação do presente registro.

O Catálogo das Aranhas do Rio Grande do Sul, de MelloLeitão (1943) faz referência a apenas alguns locais de ocorrência, nenhum deles na região central.

Também Levi, ao revisar as espécies neotropicais dos gêneros Micrathena, Alpaida, Araneus e Wagneriana (1985, 1988, 1991a e 1991b, respectivamente), quase não mencionou localidades da região central do Estado. É citada a ocorrência de Alpaida pedro em São Pedro do Sul, Alpaida trispinosa, Araneus corporosus e Araneus guttatus em Santa Maria, Micrathena spitzi em Cachoeira do Sul e Arroio do Tigre, e de Alpaida iguazu e Alpaida itauba em Arroio do Tigre. Os demais registros abrangem uma faixa que inicia em Rio Pardo, passa por Porto Alegre e arredores e alcança a região da Serra Gaúcha e Alto Uruguai , havendo ainda algumas ocorrências no oeste (Garruchos, São Borja e Quaraí) e noroeste (Bagé, Caçapava e São Gabriel) do Estado.

Para os demais gêneros, que aqui são listados, não há revisões recentes.

\section{Material e métodos}

O mapa da Fig. 1 mostra a situação, no Rio Grande do Sul, dos municípios de origem dos exemplares a partir dos quais foi composta a lista. Estão localizados na Depressão Central do Rio Grande do Sul, exceto os municípios de São Sepé, que faz parte da Campanha, e Júlio de Castilhos e Nova Palma, localizados no Planalto Médio. Os municípios de Itaara, São Martinho da Serra e Silveira Martins são antigos distritos de Santa Maria, que apenas recentemente foram emancipados e passaram a figurar em mapas; estão localizados na unidade de relevo denominada Rebordo do Planalto (Pereira et al, 1989), que é parte da zona de transição entre o Planalto Meridional Brasileiro e a Depressão Central do Rio Grande do Sul.

A maior parte do material examinado foi coletada durante trabalhos de inventário realizados em Santa Maria e Cachoeira do Sul; a forma de obtenção destes exemplares e a caracterização 
de alguns locais de coleta foram apresentados em Buss (1993) e Indrusiak \& Kotzian (1998). O material dos demais locais é produto de coletas esporádicas ou de doações. Os exemplares coletados em Cachoeira do Sul estão depositados na coleção de Arachnida do Museu de Ciências e Tecnologia (MCTP) da Pontifícia Universidade Católica do Rio Grande do Sul, cuja lista foi gentilmente fornecida pelo Curador, Dr. Arno Antonio Lise. O restante do material está depositado na coleção de aranhas do Departamento de Biologia da Universidade Federal de Santa Maria.

Como se trata de uma listagem qualitativa não foi levado em conta o número de exemplares provenientes de determinado local, sendo considerada significativa a ocorrência de um único indivíduo, desde que adulto e, portanto, passível de determinação confiável.

\section{Resultados e discussão}

As Tabelas 1 e 2 mostram a lista de espécies e seus locais de ocorrência, estes agrupados conforme as regiões fisiográficas a que pertencem.

Ao todo, foram registradas 58 espécies, vinculadas a 16 gêneros. Foi constatada pela primeira vez no Estado a ocorrência de Alpaida lanei Levi,1998, Alpaida scriba (Mello-Leitão,1940) e Araneus sicki Levi, 1991.

Em relação à lista publicada por Indrusiak \& Kotzian (1998) para Santa Maria, foram acrescidos representantes dos gêneros Kapogea, Mastophora e Neoscona, com o que o número de espécies foi ampliado para 58. Em virtude de dificuldades na determinação das espécies, ou por não haver revisões sistemáticas, ou por não terem sido obtidos exemplares adultos, os gêneros Eustala, Mangora, Mecynogea, Metepeira e Scoloderus, que figuravam na primeira lista, não foram aqui mencionados. Pela mesma razão não foram arrolados para Cachoeira do Sul os seguintes gêneros, que haviam sido listados por Buss em 1993: Bertrana, Carepalxis, Enacrosoma, Eryophora, Eustala, Hypognatha, Mangora, Metepeira, Pozonia e Scoloderus.

A aparente maior riqueza da araneofauna de Santa Maria e Cachoeira do Sul, mostrada pela figura, deve-se ao fato de só terem sido feitas coletas sistematizadas, do tipo inventário, nestes dois locais. 


\section{Considerações finais}

A presente lista constitui um considerável acréscimo ao que se conhecia anteriormente sobre as espécies de Araneidae que ocorrem na região central do Estado. Em Santa Maria e arredores, aumentou para 45 o número de espécies registradas, sendo também acrescentados 3 novos gêneros aos anteriormente conhecidos para a região. No caso de Cachoeira do Sul, as espécies arroladas passaram para 21.

Foi ampliada significativamente a área de distribuição de algumas das espécies dos gêneros neotropicais Alpaida, Araneus, Micrathena e Wagneriana, já revisados por Levi, a qual passa a abranger de maneira mais completa a região central do Estado.

Foi feito o registro, pela primeira vez, da ocorrência de Alpaida lanei Levi, 1998, Alpaida scriba (Mello-Leitão, 1940) e Araneus sicki Levi, 1991 no Rio Grande do Sul.

\section{Agradecimentos}

Ao Dr. Arno Antonio Lise (PUCRS), pela determinação da maioria dos exemplares, pela franquia dos registros da coleção de aranhas da PUCRS e, principalmente, pela orientação e apoio, sem o que este trabalho não ocorreria. A Augusto Braul, Jr. (PUCRS), pela determinação de parte dos exemplares. À Dra. Ana Beatriz Barros de Morais e à Dra. Carla Bender Kotzian, ambas da UFSM, pela revisão e sugestões ao original, além do auxílio nas coletas. Ao Dr. Ervandil Correa Costa (UFSM) pela doação do material de São Sepé. A todos os que auxiliaram nas coletas, em especial os acadêmicos da UFSM Mauro Monteiro, Juliana Dalpian e Tiago Gomes. 


\section{Bibliografia}

BERRIEL, L.P. Aranhas e insetos capturados com guarda-chuva entomológico em uma área campestre/pioneira de Santa Maria, RS, Brasil - I. Eryngium horridum Malme, 1904 (Umbelliferae). Santa Maria, RS, Universidade Federal de Santa Maria, XI + 59p,2001. Monografia de Especialização, não publicada).

BUSS, R.G. Araneofauna arborícola da região de Cachoeira do Sul, Rio Grande do Sul. Cachoeira do Sul, RS, Fundação Educacional do Vale do Jacuí / Universidade Federal de Santa Maria, 79 p., 1993. (Monografia de Especialização, não publicada).

INDRUSIAK, L.F.; KOTZIAN, C.B. Inventário das aranhas arborícolas de três regiões de Santa Maria, RS, Brasil. Rev. Ciência e Natura, Santa Maria, v.20, p. $187-214,1998$.

LEMA,T. Relações herpetofaunísticas do Rio Grande do Sul com os países vizinhos. Veritas, Porto Alegre, v. 29, n.115, p. 421 - 429, 1984.

LEVI, H. W. The spiny orb-weaver genera Micrathena and Chaetacis (Araneae: Araneidae). Bull. Mus. Comp. Zool., v. 150, n. 8, p. $429-618,1985$.

LEVI , H. W. The neotropical orb-weaving spiders of the genus Alpaida (Araneae: Araneidae). Bull. Mus. Comp. Zool., v.151, n. 7, p.365 - 487, 1988.

LEVI, H. W. a) The neotropical and mexican species of the orbweaver genera Araneus, Dubiepeira and Aculepeira (Araneae: Araneidae). Bull. Mus. Comp. Zool.,v. 152, n. 4, p. $167-315$, 1991.

b) The neotropical orb-weaver genera Edricus and Wagneriana (Araneae; Araneidae). Bull. Mus. Comp. Zool., v. 152, n. 6, p. $363-415,1991$.

MELLO-LEITÃO, C. de Catálogo das aranhas do Rio Grande do Sul. Arq. Mus. Nac., Rio de Janeiro, v. 37, p. 150 - 244, 1943.

PEREIRA, P.R.B.; GARCIA NETTO, L.R.; BORIN, C.J.A.; BARROSSARTORI, M.G. Contribuição à geografia física do Município de Santa Maria: Unidades de Paisagem. Geografia - Ensino e Pesquisa, Santa Maria, v.3, p. 37 - 68. 1989. 


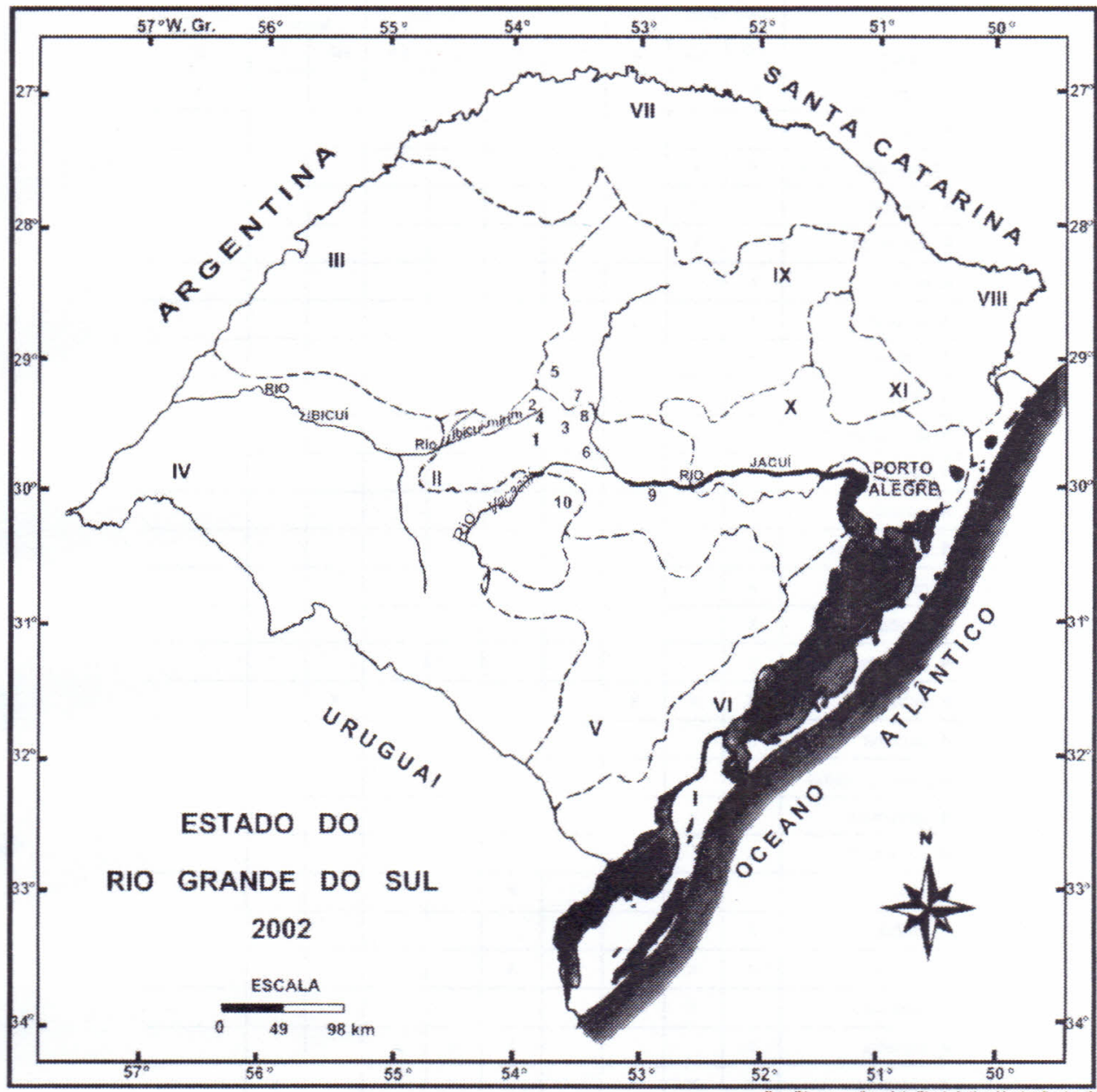

\section{LEGENDA: LOCAIS DE COUETA}

1 - Santa Maria

2. Säo Martinho da Serra 6 - Restinga Seca

3 - Silveira Martins

7. Nova Palma

4 - llaara

8. Faxinal do Soturno

5. Jillo de Castilhos

9 - Cachoeira do Sul

10 - Sảo Sepé

\section{ZONAS FISIOGRAFICAS}

1- Litoral

II. Depressão Central III - Missões

IV - Campanha

V - Serra do Sudeste

VI - Encosta do Sudeste
VII - Alto Uruguai

VIII - Campos de Cima da Serra iX - Planalto Médio

$X$ - Encosta Inferior do NE

$\mathrm{XI}$. Encosta Superior do NE

Fig. 1. Locais de ocorrência das espécies listadas 
Tabela 1. Local de ocorrência das espécies de Acacesia, Alpaida, Araneus e Argiope

\begin{tabular}{|c|c|c|c|c|c|c|c|c|c|c|}
\hline \multirow[b]{2}{*}{ Espécies } & \multicolumn{3}{|c|}{ Depressão Central } & \multicolumn{4}{|c|}{ Rebordo do Planalto } & \multicolumn{2}{|c|}{$\begin{array}{l}\text { Planalto } \\
\text { Médio }\end{array}$} & \multirow{2}{*}{$\begin{array}{c}\text { Campanha } \\
\text { SP } \\
\end{array}$} \\
\hline & SM & $\mathrm{CS}$ & RS & SE & SI & It & $\mathrm{FX}$ & NP & $\mathrm{JC}$ & \\
\hline Acacesia cornigera & $x$ & & & & $x$ & & & & & $x$ \\
\hline A. graciosa & $x$ & $x$ & & & & & & & & \\
\hline Alpaida albocincta & $x$ & $x$ & & $x$ & & & & & & \\
\hline A. alticeps & $x$ & $x$ & & & & & & & & \\
\hline A. bicornuta & $x$ & $x$ & & & & & & & & \\
\hline A. ericae & $x$ & & & & & & & & & \\
\hline A. gallardoi & $x$ & & & & & & & & & \\
\hline A. hoffmanni & $x$ & & & & & & & & & \\
\hline A. lanei & $x$ & & & & & & & & & \\
\hline A. leucogramma & & $x$ & & & & & & & & \\
\hline A. lomba & $x$ & & & & & & & & & \\
\hline A. nonoai & $x$ & & & & & & & & & \\
\hline A. quadrilorata & $x$ & & & & & & & & & \\
\hline A. rosa & $x$ & & & & & & & & & \\
\hline A. scriba & $x$ & & & & & & & & & \\
\hline A. truncata & $x$ & & & & & & & & & \\
\hline A. veniliae & $x$ & $x$ & $x$ & & & & & $x$ & & \\
\hline A. yucuma & $x$ & & & & & & & & & \\
\hline Araneus guttatus & $x$ & & & & & & & & & \\
\hline A. lathyrinus & & $x$ & & & & & & & & \\
\hline A. omnicolor & $x$ & & & & & $x$ & & & & \\
\hline A. sicki & & & & & $x$ & & & & & \\
\hline A. stabilis & $x$ & & & & & & & & & \\
\hline A. unanimus & $x$ & $x$ & & & $x$ & & & & & $x$ \\
\hline A. uniformis & & $x$ & & & & & & & & \\
\hline A. venatrix & $x$ & & & & $x$ & $x$ & & & & \\
\hline A. vincibilis & $x$ & $x$ & & & & $x$ & & & $x$ & \\
\hline A. workmani & $x$ & & & & & & & & & 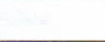 \\
\hline Argiope argentata & $x$ & $x$ & & & $x$ & & & & & $x$ \\
\hline A. trifasciata & $x$ & & & & & & & & & \\
\hline
\end{tabular}

Depressão Central: SM - Santa Maria; RS - Restinga Seca; CS - Cachoeira do Sul Rebordo do Planalto: SE- São Martinho da Serra; SI - Silveira Martins;

It - Itaara; FX - Faxinal do Soturno

Planalto Médio: NP - Nova Palma; JC - Júlio de Castilhos

Campanha: SP - São Sepé

58 Ciência \& Natura, Santa Maria, 25: 51 - 60, 2003 
Tabela 2. Locais de ocorrência das espécies de Cyclosa, Gasteracantha, Kapogea, Larinia, Mastophora, Metazygia, Micrathena, Neoscona, Ocrepeira, Parawixia, Verrucosa e Wagneriana.

\begin{tabular}{|c|c|c|c|c|c|c|c|c|c|c|}
\hline \multirow[b]{2}{*}{ Espécies } & \multicolumn{3}{|c|}{ Depressão Central } & \multicolumn{4}{|c|}{ Rebordo do Planalto } & \multicolumn{2}{|c|}{$\begin{array}{c}\text { Planalto } \\
\text { Médio }\end{array}$} & \multirow{2}{*}{$\frac{\text { Campanha }}{\text { SP }}$} \\
\hline & SM & CS & RS & SE & SI & It & $\mathrm{FX}$ & NP & $\mathrm{JC}$ & \\
\hline Cyclosa bifurcata & & $x$ & & & & & & & & \\
\hline C. diversa & $x$ & $x$ & & & & & & & & \\
\hline C. fililineata & $x$ & & & & & & & & & \\
\hline C. machadinho & & $x$ & & & & & & & & \\
\hline Gasteracantha cancriformis & $x$ & $x$ & & & & $x$ & & & $x$ & \\
\hline Kapogea alayoi & $x$ & & & & & & & & & \\
\hline Larinia montecarlo & & $x$ & & & & & & & & \\
\hline L. t-notata & $x$ & & & & & & & & & \\
\hline A. tucuman & $x$ & & & & & & & & & \\
\hline Mastophora satan & $x$ & & & & & & & & & \\
\hline Metazygia gregalis & & $x$ & & & & & & & & \\
\hline M. voluptifica & & $x$ & & & & & & & & \\
\hline Micrathena crassispina & & & & & & $x$ & & & $x$ & \\
\hline M. furcata & & $x$ & & & & & & & & \\
\hline M. furva & $x$ & & & & & & & & & \\
\hline M. lata & $x$ & & & & & & & & & \\
\hline M. nigrichelis & $x$ & & & & & $x$ & & & $x$ & \\
\hline M. rubicundula & $x$ & $x$ & & ? & & & & & & \\
\hline M. spitzi & $x$ & & & & & $x$ & & & & \\
\hline Neoscona morelli & $x$ & & & & & & & & & \\
\hline Ocrepeira gallianoae & $x$ & $x$ & & $x$ & & & & & & \\
\hline O. gnomo & & $x$ & & & & & & & & \\
\hline O. venustula & $x$ & & & & & & & & & \\
\hline Parawixia audax & $x$ & $\mathrm{x}$ & $x$ & & & $x$ & & $x$ & $x$ & $x$ \\
\hline Verrucosa meridionalis & $x$ & $x$ & & $x$ & & $x$ & & & & \\
\hline V. arenata & $x$ & & & & $x$ & $\therefore$ & & & & \\
\hline V. undecinvariolata & $x$ & $x$ & & 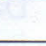 & & & $x$ & & & \\
\hline Wagneriana atuna & & $x$ & & & & & & & & . \\
\hline
\end{tabular}

Depressão Central: SM - Santa Maria; RS - Restinga Seca; CS - Cachoeira do Sul Rebordo do Planalto: SE- São Martinho da Serra; SI - Silveira Martins;

It - Itaara; FX - Faxinal do Soturno

Planalto Médio: NP - Nova Palma; JC - Júlio de Castilhos

Campanha: SP - São Sepé 
LEOCÁDIA FALKEMBERG INDRUSIAK leocadia@terra.com.br Departamento de Biologia - CCNE Universidade Federal de Santa Maria Santa Maria, RS - Brasil

Regina Gressler Buss reginabuss@ @iq.com.br Cachoeira do Sul, RS - Brasil 